\title{
Small-angle neutron-scattering studies of reentrant spin-glass behavior in Fe-Al alloys
}

Cite as: J. Appl. Phys. 99, 08H502 (2006); https://doi.org/10.1063/1.2167064

Published Online: 21 April 2006

D. Martín Rodríguez, F. Plazaola, J. J. del Val, J. S. Garitaonandia, G. J. Cuello, and C. Dewhurst

\section{ARTICLES YOU MAY BE INTERESTED IN}

Band gap estimation from temperature dependent Seebeck measurement-Deviations from

the $\left.2 \mathrm{e}|\mathrm{S}|\right|_{\max } ^{\top}$ max relation

Applied Physics Letters 106, 022112 (2015); https://doi.org/10.1063/1.4905922

Small-angle neutron scattering from magnetic correlations in Fe0.7Al 0.3

Journal of Applied Physics 52, 1732 (1981); https://doi.org/10.1063/1.329695

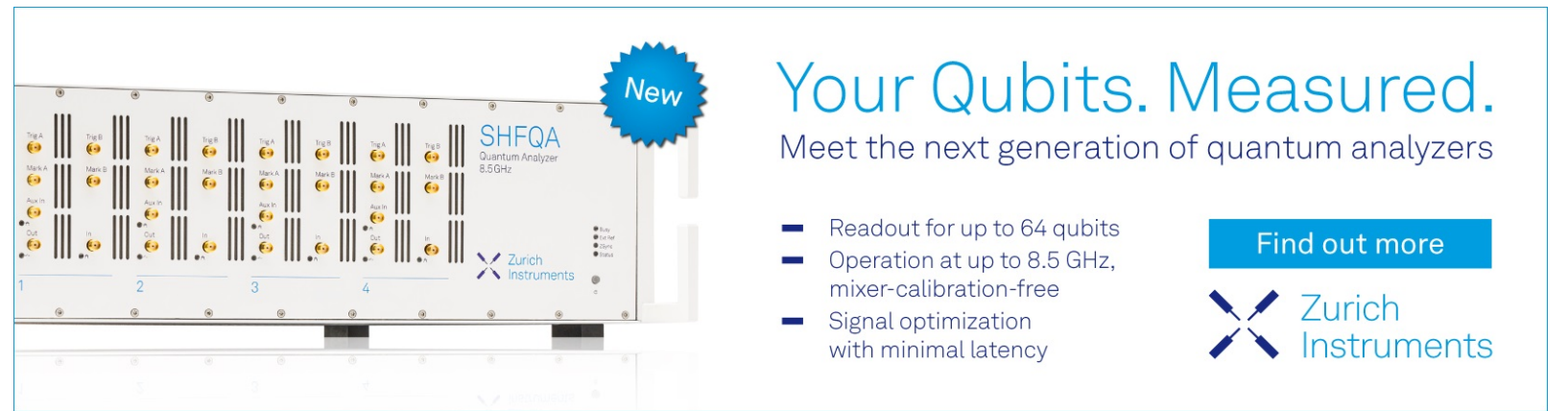




\title{
Small-angle neutron-scattering studies of reentrant spin-glass behavior in Fe-Al alloys
}

\author{
D. Martín Rodríguez ${ }^{\mathrm{a})}$ and F. Plazaola \\ Elektrika eta Elektronika Saila, UPV/EHU, 644 P. K. 48080 Bilbao, Spain
}

J. J. del Val

Departmento de Física de Materiales, Centro Mixto CSIC-UPV/EHU, UPV/EHU, Apdo. 1072,

20080 San Sebastian, Spain

J. S. Garitaonandia

Fisika Aplikatua II Saila, UPV/EHU, 644 P. K. 48080 Bilbao, Spain

G. J. Cuello and C. Dewhurst

Institute Laue Langevin, 6 rue Jules Horowitz, B. P. 156, 38042 Grenoble, France

(Presented on 1 November 2005; published online 21 April 2006)

$\mathrm{Fe}-\mathrm{Al}$ alloys around the concentration of 30 at. \% $\mathrm{Al}$ show very interesting magnetic properties. Alloys with this concentration present magnetic inhomogeneities and their evolution with temperature is also interesting because they show a reentrant spin-glass behavior at low temperatures. Small-angle neutron-scattering measurements were performed in $\mathrm{Fe}_{69.5} \mathrm{Al}_{30.5}, \mathrm{Fe}_{70} \mathrm{Al}_{30}$, and $\mathrm{Fe}_{70.5} \mathrm{Al}_{29.5}$ alloys in the temperature range of 4-300 K. Even though the change in $\mathrm{Fe}$ content is very small, the response variation is quite large. The results show that the samples have magnetic inhomogeneities that evolve with temperature. Only in the Fe-richest sample the random-field model can be applied. () 2006 American Institute of Physics.

[DOI: $10.1063 / 1.2167064]$

$\mathrm{Fe}-\mathrm{Al}$ alloy system is very interesting mainly for the controversies it presents about magnetic order. Indeed, for concentrations around 30 at. \% $\mathrm{Al}$ show very interesting magnetic properties. For instance, the magnetization of the $\mathrm{Fe}_{70} \mathrm{Al}_{30}$ alloy decreases with temperature from the paramagnetic state to a ferromagnetic one at Curie temperature $\left(T_{C}\right)$ and then, below $T_{C}$, to another one at the inversion temperature $\left(T_{i}\right)$, where ferromagnetic long-range order (LRO) breaks down into magnetic clusters upon cooling. ${ }^{1}$ Finally, below $T_{i}$, there is another transition to spin-glass phase at the freezing temperature $\left(T_{g}\right)$. Therefore, the appearance of a spin-glass state at a temperature lower than that of the LRO indicates that $\mathrm{Fe}_{70} \mathrm{Al}_{30}$ is a reentrant spin-glass (RSG) system. ${ }^{1}$ By neutron diffuse scattering Cable et $a .^{2}$ found that this alloy has a magnetic cluster structure with an average size of $25 \AA$ which develops with temperature. Furthermore, Bao et al. ${ }^{3}$ showed that the ferromagnetic state and paramagneticlike state coexist as a mixed state, claiming that the origin of this magnetic behavior is due to compositional inhomogeneities occurring in the nonstoichiometric D03 structure.

Several small-angle neutron-scattering (SANS) experiments have been performed in $\mathrm{Fe}_{70} \mathrm{Al}_{30}$ alloy, ${ }^{2-5}$ but only one SANS experiment has been performed on a different concentration close to 30 at. $\% \mathrm{Al}\left(\mathrm{Fe}_{71.5} \mathrm{Al}_{28.5}\right){ }^{6}$ According to the experiments by Cable et al. ${ }^{2}$ the data do not show any anomaly neither at $T_{i}$ nor at $T_{g}$ transition temperatures. It has to be remarked that their data were taken over a relatively large $Q$ range from 0.1 to $0.5 \AA^{-1}$. In later works ${ }^{4,5}$ the re-

${ }^{\text {a) Electronic mail: dmr@we.lc.ehu.es }}$ sults showed well-defined singularities in the thermal evolution of the SANS intensities at $T_{C}$ and at $T_{i}$. However, the singularity at $T_{g}$ is less clear. The discrepancies between the Cable et al. report and the following ones stem from the difference in the measured $Q$ range. Suzuki et al. ${ }^{6}$ performed SANS experiments on the $\mathrm{Fe}_{71.5} \mathrm{Al}_{28.5}$ alloy in a wider range than the works by Child ${ }^{4}$ and Motoya et al. ${ }^{5}$ In the small $Q$ range below $0.05 \AA^{-1}$ the peak corresponding to the phase transition at $T_{g}$ appears, unlike in the other mentioned works. It has to be taken into account that the magnetization curves in the two previously cited compositions behave in a very different way; ${ }^{7}$ then a direct comparison between the results obtained by SANS in the two $\mathrm{Fe}-\mathrm{Al}$ compositions is not straightforward.

In order to get a better insight on the RSG phenomena in $\mathrm{Fe}-\mathrm{Al}$ alloys and with the aim of filling the gap in compositions studied by diffuse neutron scattering, we present SANS and magnetization measurements from $4 \mathrm{~K}$ up to room temperature (RT) in $\mathrm{Fe}_{69.5} \mathrm{Al}_{30.5}, \mathrm{Fe}_{70} \mathrm{Al}_{30}$, and $\mathrm{Fe}_{70.5} \mathrm{Al}_{29.5}$ alloys.

$\mathrm{Fe}_{69.5} \mathrm{Al}_{30.5}, \mathrm{Fe}_{70} \mathrm{Al}_{30}$, and $\mathrm{Fe}_{70.5} \mathrm{Al}_{29.5}$ alloys were prepared by induction melting and cast into ingots in an Ar atmosphere; then a powder was obtained by mechanical crushing. In order to obtain D03 long-range ordered samples, a long-time heat treatment was applied. Powders were annealed at about $1200 \mathrm{~K}$ for $2 \mathrm{~h}$ in order to homogenize them. After, they were cooled down at a rate of $20 \mathrm{~K} / \mathrm{h}$ up to 825 $\mathrm{K}$ and from this temperature down to $625 \mathrm{~K}$ they were cooled at a rate of $1 \mathrm{~K} / \mathrm{h}$. Finally they were left to cool down up to RT.

Neutron-scattering experiments were conducted at the small-angle scattering diffractometer D11 at the Institut Laue Langevin (Grenoble, France). Samples were placed in a va- 


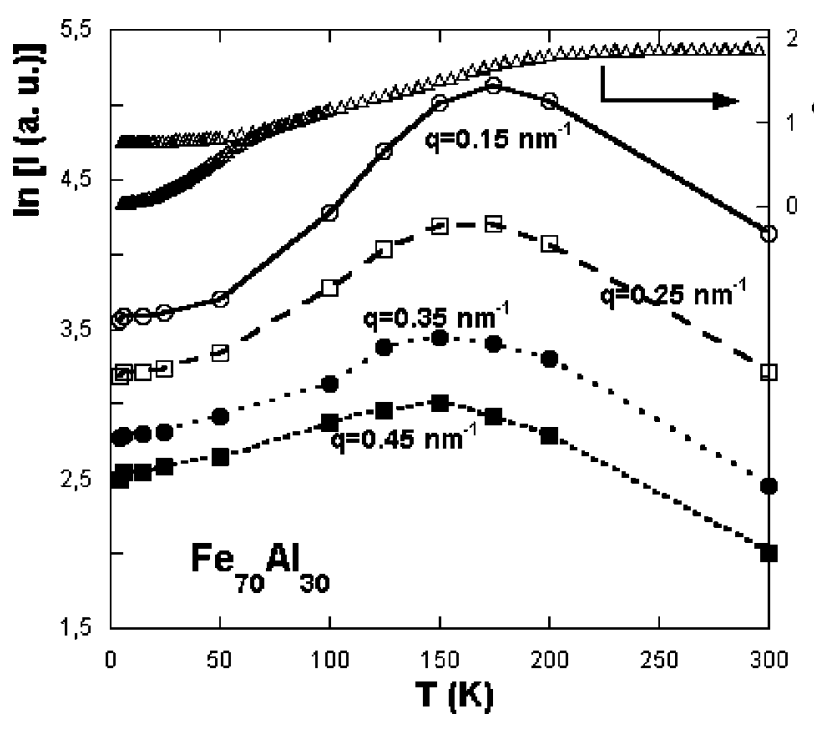

FIG. 1. ZFC-FC curve (triangles) and the evolution of the intensity of SANS spectrum with temperature at various $Q$ of $\mathrm{Fe}_{70} \mathrm{Al}_{30}$ alloy. Lines are drawn to guide the eye.

nadium cell in a standard orange cryostat, which allowed us to measure in the 4-300 K range. Samples were measured with a neutron wavelength of $4.51 \AA$ and in a range of $0.04 \leqslant Q \leqslant 0.31 \AA^{-1}$. Water and Cd samples and empty cell were used to measure the detector efficiency and the background contribution. Data collected on the two-dimensional detector for each sample were azimuthally averaged, corrected for background and absorption, and normalized to the absolute cross section.

Zero-field cooling-field cooling (ZFC-FC) where performed with a magnetic field of 60 Oe in a superconducting quantum interference device(SQUID) magnetometer (Quantum Design MPMS).

The raw data corresponding to all the samples can be fitted properly using the Guinier approximation only for $Q$ $>0.05 \AA^{-1}$. The different behavior of the SANS intensity with $Q$ above and below $0.05 \AA^{-1}$ in all the studied samples is in agreement with the different thermal evolution of the SANS data reported by Suzuki et $a l .{ }^{6}$ As the Guinier approximation is related to density fluctuations occurring in the sample, ${ }^{8}$ we have subtracted that contribution in all the data.

Figure 1 shows ZFC-FC measurements together with plots of the temperature dependence of the scattered neutron intensity for four-momentum transfers in the low $Q$ range $\left(0.01 \leqslant Q \leqslant 0.05 \AA^{-1}\right)$. It is interesting to notice the presence of a well-defined peak at about $175 \mathrm{~K}$ in the $\mathrm{Fe}_{70} \mathrm{Al}_{30}$ alloy already reported by Motoya et al., ${ }^{5}$ i.e., at $T_{i}$. The peak shifts a bit to lower temperatures with increasing the momentum transfer. The shape of this peak is reminiscent of what could be expected for a broad magnetic transition comprising a distribution of transition temperatures. However, in $T_{g}$ no features are observed.

In the case of $\mathrm{Fe}_{70.5} \mathrm{Al}_{29.5}$ alloy, the behavior shown by the momentum transfer $Q$ shows a similar peak to the one observed in $\mathrm{Fe}_{70} \mathrm{Al}_{30}$ (see Fig. 2). However, the peak shifts noticeably to lower temperatures with increasing momentum transfer (between 50 and $150 \mathrm{~K}$ ). This alloy does not show

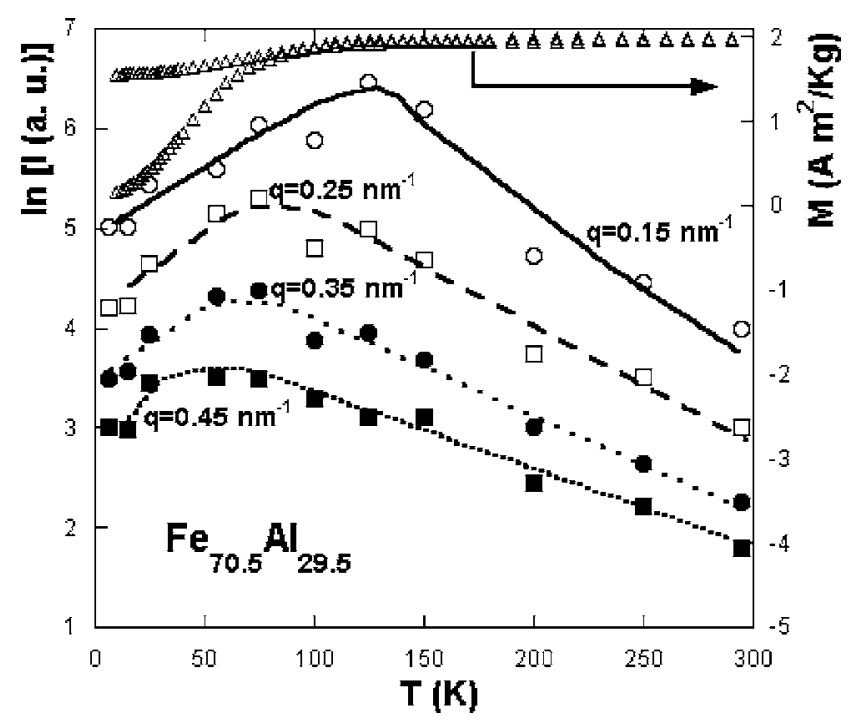

FIG. 2. ZFC-FC curve (triangles) and the evolution of the intensity of SANS spectrum with temperature at various $Q$ of $\mathrm{Fe}_{70.5} \mathrm{Al}_{29.5}$ alloy. Lines are drawn to guide the eye.

any inversion temperature, which indicates that the transition to the spin-glass state is from the ferromagnetic one.

The behavior presented by the $\mathrm{Fe}_{69.5} \mathrm{Al}_{30.5}$ alloy is completely different to the previous two ones. The momentum transfer does not present any peak only in the smallest $Q$ value presented in Fig. 3. The peak present for the larger $Q$ values of Fig. 3 appears at temperatures close to the change of slope of the corresponding magnetization curve (see ZFC-FC in Fig. 3), but not at $T_{g}$. The scattering intensity decreases abruptly for the four $Q$ values shown at temperatures of about $10 \mathrm{~K}$, well below $T_{g}$.

In the literature the experimental data have been fitted with different scattering functions such as Lorentzian, squared Lorentzian, ${ }^{4}$ power law, ${ }^{5}$ and the sum of the Lorentzian and the squared-Lorentzian ${ }^{6}$ forms. Lorentzian structure factors are normally obtained when temperature is the prin-

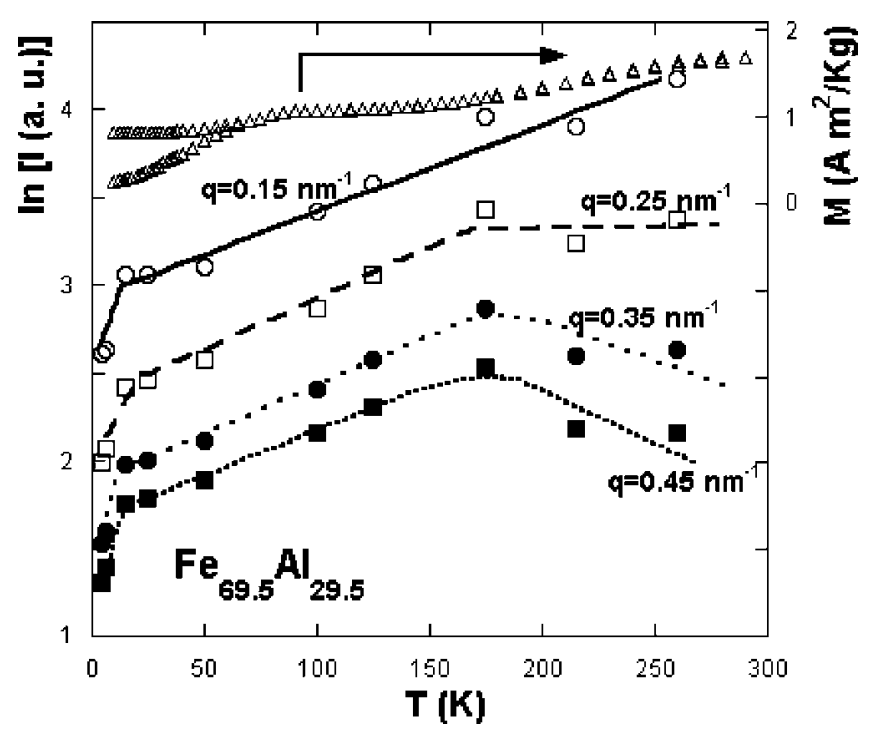

FIG. 3. ZFC-FC curve (triangles) and the evolution of the intensity of SANS spectrum with temperature at various $Q$ of $\mathrm{Fe}_{69.5} \mathrm{Al}_{30.5}$ alloy. Lines are drawn to guide the eye. 
cipal source of disorder and the presence of small random fields is taken into account by a structure factor sum of a Lorentzian and a squared Lorentzian. In order to compare the behavior of the scattering intensity with temperature in all the studied samples, we have fitted the scattering data (after subtracting the Guinier contribution) with the pseudoLorentzian function $A /\left(\kappa^{2}+Q^{\beta}\right)$, where $\beta=2$ corresponds to a Lorentzian (Ornstein-Zernike form) and $\kappa$ is the inverse correlation length. $\mathrm{Fe}_{70.5} \mathrm{Al}_{29.5}$ alloy can be fitted with a Lorentzian scattering function $(\beta=2)$ in the whole temperature range. This is in agreement with the analyses of the SANS data for $Q<0.05 \AA^{-1}$ by Suzuki et al. ${ }^{6}$ The ZFC-FC curves of the sample studied by Suzuki et al. and the one presented in Fig. 2 are similar; then the transition from the ferromagnetic state to the spin-glass one is direct. The behavior of $\kappa$, the inverse correlation length (not shown), is what could be expected in a ferromagnetic to spin-glass transition, nonzero only up to $T_{g}$. Decreasing the amount of $\mathrm{Fe}$ content a bit, $\mathrm{Fe}_{70} \mathrm{Al}_{30}$ alloy shows that the scattering data can be fitted with a Lorentzian function and $\kappa=0$ only down to about $170 \mathrm{~K}$, i.e., just down to $T_{i}$. At low temperatures $\beta$ goes down to a value of 1.2. Decreasing further the Fe content, $\mathrm{Fe}_{69.5} \mathrm{Al}_{30.5}$ alloy, a Lorentzian function can be used only to fit the data close to room temperature; however, $\kappa$ value is small but nonzero. It is in agreement with the fact that the ZFC-FC curve (see Fig. 3) does not reach the $T_{i}$ transition below room temperature. In the samples with larger Fe content the lower the $Q$ value the closer the peak position to $T_{i}$. Therefore, the lack of maximum for the lowest $Q$ values in Fig. 3 may indicate that $T_{i}$ transition has not been reached at the maximum measured temperature. Moreover, the shift on peak position suggests that the largest characteristic lengths are at the origin of the $T_{i}$ transition in each alloy.

We have also tried to fit the scattering data with the sum of Lorentzian and a squared-Lorentzian. In the case of $\mathrm{Fe}_{70.5} \mathrm{Al}_{29.5}$ alloy and close to room temperature the squaredLorentzian contribution is very small, but it increases with decreasing temperature, and at $4 \mathrm{~K}$ its contribution is very close to the Lorentzian one. Therefore, this behavior indicates that the RSG occurs in this alloy following the randomfield model. ${ }^{9}$ However, in the case of the other two samples, present model does not give a proper fit for temperatures below $T_{i}$.

SANS measurements indicate that the studied $\mathrm{Fe}-\mathrm{Al}$ alloys present magnetic inhomogeneities that vary drastically with Fe content for alloys around 30 at. \% $\mathrm{Al}$ and with temperature. Indeed, SANS results indicate that the temperature evolution of the magnetic inhomogeneities is the cause of the behavior presented by the magnetization measurements.

Summarizing, we have performed SANS and magnetic measurements for $\mathrm{Fe}_{69.5} \mathrm{Al}_{30.5}, \mathrm{Fe}_{70} \mathrm{Al}_{30}$, and $\mathrm{Fe}_{70.5} \mathrm{Al}_{29.5}$ alloys and there are three main conclusions.

- The behavior of the SANS intensity with $Q$ above and below $0.05 \AA^{-1}$ in all the studied samples is different. For $Q>0.05 \AA^{-1}$ the data follow a Guinier function. However, below $0.05 \AA^{-1}$ the fit of the data depends on composition and temperature.

- The alloy with the largest Fe content $\left(\mathrm{Fe}_{70.5} \mathrm{Al}_{29.5}\right)$ is the only one that follows the Ornstein-Zernike form in the temperature range of 4-300 K. For Al contents larger than 29.5 at. \% the Ornstein-Zernike form fails at low temperatures and the temperature at which it starts to fail increases with $\mathrm{Al}$ content. The SANS results indicate that random-field model can be applied for the Fe-richest alloy, but it is not so straightforward for larger $\mathrm{Al}$ content.

- In all samples magnetic inhomogeneities are detected. The presence of magnetic inhomogeneities induces the coexistence of ferromagnetic order and paramagneticlike disorder at different length scales which evolve with temperature and are responsible for the behavior observed in magnetization measurements.

This work was undertaken under Project Nos. MAT2002-4087-C02-01 and UPV 224.310-14553/2002. One of the authors (D.M.R.) wishes to thank UPV-EHU for the fellowship.

${ }^{1}$ S. Mitsuda, H. Yoshizawa, and Y. Endoh, Phys. Rev. B 45, 9788 (1992).

${ }^{2}$ J. W. Cable, L. David, and R. Parra, Phys. Rev. B 16, 1132 (1977).

${ }^{3}$ W. Bao, S. Raymond, S. M. Shapiro, K. Motoya, B. Fåk, and R. W. Erwin, Phys. Rev. Lett. 82, 4711 (1999).

${ }^{4}$ H. R. Child, J. Appl. Phys. 52, 1732 (1981).

${ }^{5}$ K. Motoya, S. M. Shapiro, and Y. Muraoka, Phys. Rev. B 28, 6183 (1983).

${ }^{6}$ J. Suzuki, Y. Endoh, M. Arai, M. Furusaka, and H. Yoshizawa, J. Phys. Soc. Jpn. 59, 718 (1990).

${ }^{7}$ R. D. Shull, H. Okamoto, and P. A. Beck, Solid State Commun. 20, 863 (1976).

${ }^{8}$ E. A. Porai-Koskits, J. Non-Cryst. Solids 25, 86 (1977).

${ }^{9}$ G. Aeppli, S. M. Shapiro, R. J. Birgeneau, and H. S. Chen, Phys. Rev. B 28, 5160 (1983). 